\title{
QUANTITATIVE ESTIMATIONS OF THE \\ ANOMALOUS PLASMA DIFFUSION \\ IN AN ACTIVE REGION*
}

\author{
M. KOPECKÝ** and G.V. KUKLIN
}

(Siberian Institute of Terrestrial Magnetism, Ionosphere and Radio Propagation, Academy of Sciences, Irkutsk, U.S.S.R.)

In some recent papers the interdependence of the gas and magnetic-field motions in the solar atmosphere was considered. Some results indicate the occurrence of gas motion along the magnetic-field lines combined with motion of the field line, but sometimes we have to assume an obvious gas motion across the magnetic-field lines. As one of the possible mechanisms explaining this fact the anomalous plasma diffusion may be proposed.

In this paper we attempt to estimate the Böhm-diffusion effect. We are inconsistent in a certain sense using the Böhm-diffusion theory for a fully ionized plasma (Galeev et al., 1963) in the case of a partly ionized plasma. In an unbounded medium the Bohmdiffusion coefficient has a maximal value

$$
D_{\perp M}^{B}=\frac{c k T}{2 \pi e H}=\frac{6.91 \times 10^{6}}{9 H}
$$

and in a system with longitudinal and transversal sizes $L$ and $\rho L$ correspondingly it is

$$
D_{\perp}^{B}=\begin{array}{cc}
c k T H_{*} \\
2 \pi e H H
\end{array}
$$

where

$$
H_{*}=e^{c}\left(\frac{v_{\mathrm{ei}} m_{\mathrm{i}} m_{\mathrm{e}} k T_{\mathrm{e}}}{\rho^{4} L^{2}}\right)^{1 / 3}=4.09 \times 10^{5}\left(\begin{array}{c}
\mu_{\mathrm{i}} r_{\mathrm{e}}^{2} P_{\mathrm{e}} \\
\rho^{4} L^{2} \sqrt{\vartheta}
\end{array}\right)^{1 / 3} .
$$

The Böhm diffusion is effective when it is not masked by the classical diffusion

$$
D_{\perp M}^{B}>D_{\perp}^{C} \text {. }
$$

Taking into account both the low ionization degree plasma and the almost fully ionized one we must write down in a rough approximation

$$
D_{\perp}^{C} \approx D_{\perp}^{A}+D_{\perp}^{L},
$$

* Presented by G.V. Kuklin.

** On leave from the Astronomical Institute of the Czechoslovak Academy of Sciences, Ondřejov. 
where $D_{\perp}^{A}$ is the ambipolar diffusion coefficient in the fully ionized plasma, and $D_{\perp}^{L}$ is the classical diffusion coefficient in the low ionized plasma. Then a critical value of the magnetic field strength $H_{0}$, when the relation (3) is correct, is

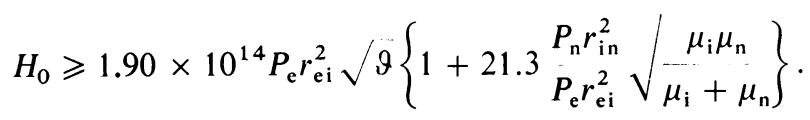

Using the data given in Appendix $C$ of the paper by Zwaan (1965) we have computed $\log H_{0}$ in gausses (Table 1), $\log H_{*}\left(\rho^{4} L^{2}\right)^{1 / 3}$ (Table 2), and $\log D_{\perp M}^{B}$ (Table 3) for some values of $\vartheta$ and $P_{g}$. We have taken $r_{\mathrm{in}}^{2}=10^{-15} \mathrm{~cm}^{2}$ and the values $D_{\mathrm{L}}^{B}$ Max are computed for $H=H_{0}$ according to Equation (1). All designations are the same as in our previous papers (Kuklin, 1966; Kopecký and Kuklin, 1966, 1967).

\section{Table 1}

$\log H_{0}$

$\begin{array}{ccccc}\log P_{g} / \vartheta & 0.8 & 1.1 & 1.4 & 1.7 \\ 3.0 & 3.50 & 3.70 & 3.75 & 3.79 \\ 4.5 & 5.04 & 5.20 & 5.25 & 5.29 \\ 6.0 & 6.60 & 6.70 & 6.75 & 6.79\end{array}$

Table 2

$\log H_{*}\left(\rho^{4} L^{2}\right)^{1 / 3}$

$\begin{array}{ccccc}\log P_{g} / \vartheta & 0.8 & 1.1 & 1.4 & 1.7 \\ 3.0 & 1.58 & 1.50 & 1.39 & 1.23 \\ 4.5 & 1.90 & 1.91 & 1.73 & 1.62 \\ 6.0 & 2.30 & 2.25 & 2.10 & 1.94\end{array}$

Table 3

$\log D_{\perp}^{B}$

$\begin{array}{ccccc}\log P_{g} / \vartheta & 0.8 & 1.1 & 1.4 & 1.7 \\ & & & & 2.82 \\ 3.0 & 3.44 & 3.10 & 2.94 & 1.32 \\ 4.5 & 1.90 & 1.60 & 1.44 & -0.18 \\ 6.0 & 0.34 & 0.10 & -0.06 & \end{array}$

Looking through the tables we can be convinced that within the physical parameter range of the sunspotss the photosphere, the low chromosphere, and the faculae, the Böhm-diffusion effect is negligible for the macroscopic regions. It is caused firstly by too high critical values of $H_{0}$, which seldom occur in reality; secondly by too low values of $D_{\perp}^{B}$, which can not explain usually observed discrepancies; and thirdly by 
too low values of $H_{*}\left(\rho^{4} L^{2}\right)^{1 / 3}$. One can see that the filament in the sunspot penumbra with $\rho \approx 10^{-3}$ and $H_{*} \sim 1$ gauss cannot be longer than tens of kilometers. So the Böhm diffusion would have some meaning for systems of such sizes only, but it does not exclude the possibility for other anomalous diffusion types to be essential.

\section{References}

Galeev, A. A., Moiseev, S.S., Sagdeev, R.Z.(1963) ' Preprint, Novosibirsk.

Kopecký, M., Kuklin, G.V. (1966) Bull. astr. Inst. Csl., 17, 45.

Kopecký, M., Kuklin, G.V. (1968) Solar Phys., 4, in press.

Kuklin, G.V. (1966) Resultaty nabl. i issled. v period. MGSS, 1, 17.

Zwaan, C. (1965) Rech. astr. Obs. Utrecht, 17, 4. 